\title{
Factors Associated with Duration of Intercostal Chest Drainage in Patients with Primary Spontaneous Pneumothorax and the Role of Pulmonary Rehabilitation
}

\author{
Puthachad Namwaing' \\ Seksan Chaisuksant ${ }^{\prime}$ \\ Rakfan Sawadpanich ' \\ Thitima Anukunananchai' \\ Akkaranee Timinkul $^{2}$ \\ Waraporn Sakaew ${ }^{3}$ \\ Bundit Sawunyavisuth ${ }^{4}$ \\ Watchara Boonsawat ${ }^{2}$ \\ Sittichai Khamsai ${ }^{2}$ \\ Kittisak Sawanyawisuth (iD) ${ }^{2}$ \\ 'Khon Kaen Regional Hospital, Khon \\ Kaen, Thailand; ${ }^{2}$ Department of \\ Medicine, Faculty of Medicine, Khon \\ Kaen University, Khon Kaen, Thailand; \\ ${ }^{3}$ Department of Anatomy, Faculty of \\ Medicine, Khon Kaen University, Khon \\ Kaen, Thailand; ${ }^{4}$ Department of \\ Marketing, Faculty of Business \\ Administration and Accountancy, Khon \\ Kaen University, Khon Kaen, Thailand
}

Correspondence: Sittichai Khamsai; Kittisak Sawanyawisuth

Department of Medicine, Faculty of

Medicine, Khon Kaen University, 123

Mitraparp Road, Khon Kaen, 40002,

Thailand

Tel +66-43-363664

Fax +66-43-348399

Email sittikh@kku.ac.th;

kittisak@kku.ac.th
Purpose: Primary spontaneous pneumothorax (PSP) is an urgent/emergency condition. Treatment with intercostal chest drainage (ICD) is necessary, particularly in symptomatic patients or those with tension. A previous study found that systematic breathing exercise significantly reduced ICD duration when compared with controls. This study aimed to evaluate if pulmonary rehabilitation can reduce the duration of ICD treatment in patients with PSP.

Patients and Methods: This was a retrospective study of patients diagnosed with PSP treated with ICD. Duration of ICD treatment was recorded from patients' medical charts. Factors associated with ICD duration were calculated using linear regression analysis.

Results: There were 66 patients who met the study criteria, with average (SD) age and body mass index of $31.68(13.53)$ years and $20.94(2.72) \mathrm{kg} / \mathrm{m}^{2}$. The majority of the patients were male $(72.73 \%)$, and average (SD) duration of ICD treatment was 9.90 (7.83) days. Three factors remained in the final model: body mass index, systolic blood pressure, and recurrent PSP. Two factors were independently associated with longer ICD duration: systolic blood pressure and recurrent PSP, with adjusted coefficients of 0.21 ( $\mathrm{p}$ value 0.041 ) and 7.69 (p value 0.039), respectively. Pulmonary rehabilitation was not included in the final model.

Conclusion: Patients with a history of recurrent PSP or high systolic blood pressure at presentation may require longer ICD duration. Pulmonary rehabilitation was not associated with the duration of ICD treatment.

Keywords: systolic blood pressure, recurrent pneumothorax, body mass index

\section{Introduction}

Primary spontaneous pneumothorax (PSP) - defined as air in the pleural space without any identifiable cause - is an urgent/emergency condition. It is more common in males than in females (18-28 cases vs $2-6 / 100,000$ population). ${ }^{1}$ Smoking 22 cigarettes/day increases the risk of PSP by 22 times in men and ninefold in women. ${ }^{2}$ A recent study also found that PSP may be associated with weather, particularly temperature (odds ratio of 1.97; p 0.018), humidity (OR 1.58; p 0.043), and duration of sunshine (odds ratio $2.26 ; \mathrm{p} 0.012){ }^{3}$ At least $11 \%$ of patients may have recurrent episodes of PSP within five years. ${ }^{4}$ However, the recurrence rate may be as high as $26.5 \%$ within one year or up to $54 \%$ over five years. ${ }^{5-7}$

Although there are several treatment options available for PSP, such as observation, intercostal chest drainage (ICD), or surgical intervention, there have been few 
randomized controlled trials in which two or more of these options were compared head-to-head. ${ }^{4}$ One randomized controlled trial found that conservative treatment may be non-inferior to ICD treatment. ${ }^{8}$ However, $15 \%$ of patients with PSP must still undergo pleural drainage via ICD. ${ }^{4}$ Pulmonary rehabilitation may also be beneficial for PSP. Deep breathing exercises with or without incentive spirometer have been shown to improve lung parameters including vital capacity $(72.12 \%$ to $86.24 \%)$, force vital capacity, force expiratory volume in one second, and maximal voluntary ventilation. ${ }^{9}$ Additionally, one study found that systematic breathing exercise significantly reduced ICD duration compared with controls (4.80 vs 6.85 days; $\mathrm{p}=0.002) .{ }^{10}$ However, the study was conducted on only 20 patients, and the results may only be applicable to those specific to breathing exercise used. This study thus aimed to evaluate whether any of the various pulmonary rehabilitation methods used in clinical practice can reduce the duration of ICD treatment in patients with PSP.

\section{Methods}

This was a retrospective, cohort study conducted at Khon Kaen Regional Hospital. The inclusion criteria were diagnosis with PSP, treatment with ICD, and available records of ICD duration. Patients with secondary pneumothorax, who were treated with surgical intervention, whose clinical data were unavailable, and who were pregnant were excluded. The study period was between 1997 and 2018. This study was a part of primary spontaneous pneumothorax project.

Eligible patients' charts were reviewed to assess baseline characteristics, physical signs, laboratory tests, and physical therapy modalities. The primary outcome of the study was the duration of ICD treatment. Baseline characteristics included age, sex, co-morbid disease, history of smoking/alcohol consumption, and history of recurrent PSP, while physical signs consisted of vital signs and oxygen saturation at the time of PSP diagnosis. Pain was also evaluated using a visual analog scale (VAS) at the time of diagnosis. Laboratory tests included complete blood count, glucose, blood urea nitrogen, serum albumin, serum cholesterol, serum alanine aminotransferase (ALT), serum aspartate transaminase (AST), and chest x-ray findings related to PSP. The physical therapy modalities performed depended on the patient's condition.

\section{Statistical Analyses}

Descriptive statistics were used to calculate studied factors. Numerical variables were expressed as mean (SD), while number (percentage) was used for categorical variables. Factors associated with the duration of ICD treatment were calculated using linear regression analysis. Univariate linear regression was used to compute the unadjusted coefficient, 95\% confidence interval, and $\mathrm{p}$ value for each factor. Potential factors with $\mathrm{p}$ values of less than 0.20 (clinical significance) were included in the subsequent stepwise multivariate linear regression analysis. Factors with $\mathrm{p}$ values less than 0.25 were retained in the final model. Those with multicollinearity were excluded from the model. All statistical analyses were performed using STATA (College Station, Texas, USA).

\section{Results}

There were 66 patients with PSP who met the study criteria. The average (SD) age and body mass index of the patients were 31.68 (13.53) years and $20.94(2.72) \mathrm{kg} /$ $\mathrm{m}^{2}$. The majority were male $(72.73 \%)$ with an average VAS pain score of 4.32/10 (SD 3.19). Other baseline characteristics are shown in Table 1. Table 2 shows patients' laboratory results. Pneumothorax larger than $2 \mathrm{~cm}$ was found in $64.00 \%$ of patients, mostly on the right side $(69.70 \%)$. The common physical therapy modalities used to treat the patients are shown in Table 3, the most common of which being breathing exercise (80.36).

The average (SD) duration of ICD treatment was 9.90 (7.83) days. Three factors remained in the final model: body mass index, systolic blood pressure, and recurrent PSP. Unadjusted coefficients of factors included in the model are shown in Table 4, while the adjusted coefficients are shown in Table 5. Two factors were independently associated with longer duration of ICD: high systolic blood pressure and recurrent PSP with adjusted coefficients of 0.21 ( $p$ value 0.041) and 7.69 ( $p$ value 0.039). Pulmonary rehabilitation did not remain in the final model.

\section{Discussion}

Two factors were significantly related to the longer duration of ICD treatment in patients with PSP: high systolic blood pressure and recurrent PSP (Table 5).

The mechanisms underlying the association between systolic blood pressure and ICD treatment duration remain unclear. We postulate that it may be related to pain. Acute pain signals from tissue trauma markedly increase muscle sympathetic activity. Nociceptive stimuli also stimulate the locus coeruleus resulting in excretion of norepinephrine and corticotropin-releasing hormones, which increase blood 
Table I Baseline Characteristics of Patients with Primary Spontaneous Pneumothorax Treated by Intercostal Drainage $(n=66)$

\begin{tabular}{|l|l|}
\hline Factors & Mean (SD) or Number (\%) \\
\hline Age, years & $31.68(13.53)$ \\
Male sex & $48(72.73)$ \\
\hline Co-morbid disease & \\
Diabetes & $2(3.03)$ \\
Hypertension & $4(13.56 .066)$ \\
Allergic rhinitis & $2(3.03)$ \\
Asthma & $2(3.39)$ \\
Smoking & $32(48.48)$ \\
Alcohol consumption & $29(44.62)$ \\
Recurrent PSP & $19(28.79)$ \\
Body mass index, $\mathrm{kg} / \mathrm{m}^{2}$ & $20.94(2.72)$ \\
Body temperature, ${ }^{\circ} \mathrm{C}$ & $37.05(0.66)$ \\
Respiratory rate, tpm & $21.10(2.03)$ \\
Heart rate, bpm & $86.78(14.91)$ \\
Systolic blood pressure, $\mathrm{mmHg}$ & $128.67(19.23)$ \\
Diastolic blood pressure, $\mathrm{mmHg}$ & $77.5 \mathrm{I}(12.58)$ \\
Oxygen saturation, \% & $97.56(2.28)$ \\
Visual analogue scale & $4.32(3.19)$ \\
\hline
\end{tabular}

pressure. ${ }^{11}$ High systolic blood pressure may be associated with more severe tissue damage, which causes greater pain and results in longer ICD duration. Even though a visual analogue scale was put in the model, it was not retained in the final model. These results may indicate that there might be

Table 2 Laboratory Results of Patients with Primary Spontaneous Pneumothorax Treated by Intercostal Drainage $(n=66)$

\begin{tabular}{|l|l|}
\hline Factors & Mean (SD) or Number (\%) \\
\hline Hemoglobin, g/dL & $13.74(1.92)$ \\
Hematocrit, \% & $40.37(6.06)$ \\
White blood cells, $\times 10^{3} / \mathrm{mm}^{3}$ & $9.88(3.69)$ \\
PMN, \% & $69.42(1 \mathrm{I} .3 \mathrm{I})$ \\
Lymphocytes, \% & $21.89(9.98)$ \\
Platelet, $\times 10^{6} / \mathrm{mm}^{3}$ & $273(86)$ \\
Glucose, $\mathrm{mg} / \mathrm{dL}$ & $98.81(14.48)$ \\
Blood urea nitrogen, $\mathrm{mg} / \mathrm{dL}$ & $12.05(12.49)$ \\
Creatinine, $\mathrm{mg} / \mathrm{dL}$ & $0.85(0.35)$ \\
Serum albumin, g/dL & $4.13(0.46)$ \\
Cholesterol, $\mathrm{mg} / \mathrm{dL}$ & $163.95(39.67)$ \\
ALT, U/L & $32.63(39.16)$ \\
AST, U/L & $39.45(63.75)$ \\
Size $>2 \mathrm{~cm}$ & $16(64.00)$ \\
Right side & $46(69.70)$ \\
Tension pneumothorax & 0 \\
\hline
\end{tabular}

Abbreviations: ALT, alanine aminotransferase; AST, aspartate transaminase.
Table 3 Related Physical Therapy Modalities in Patients with Primary Spontaneous Pneumothorax Treated by Intercostal Drainage $(n=66)$

\begin{tabular}{|l|c|}
\hline Physical Therapy Modalities & Number (\%) \\
\hline Any modality & $57(86.36)$ \\
Ward/Home Program & $56(84.85)$ \\
Breathing exercise & $53(80.30)$ \\
Chest mobilization & $49(74.24)$ \\
Pulmonary exercise & $28(42.42)$ \\
Ambulation training & $25(37.88)$ \\
Pleurisy exercise & $12(18.18)$ \\
\hline
\end{tabular}

other contributing factors to explain the association between systolic blood pressure and duration of ICD. Note that diastolic blood pressure was not included in the model due to collinearity with the systolic blood pressure. Systolic blood pressure had a coefficient of 0.20 , indicating that for every 1 $\mathrm{mmHg}$ increase in systolic blood pressure, ICD duration increased by 0.20 days. Although this association is statistically significant, further studies are necessary to determine its clinical significance.

Even though recurrent PSP may be associated with treatment options, ${ }^{12}$ at least two factors were shown to be associated with recurrent PSP. ${ }^{13,14}$ Occurrence of PSP at home and presence of bubbles on a chest $\mathrm{CT}$ scan increased the risk of recurrent PSP by 1.43 times $(p=0.025)$ and 3.26 times $(p<0.008)$, respectively. These two factors may indicate scarring or a large pneumothorax, both of which can result in longer ICD treatment.

Although body mass index may be an indicator of PSP and remained in the final model, ${ }^{1}$ it was not related to the duration of ICD treatment (Table 5). Rehabilitation modality was also unrelated and was not included in the final model. One possible explanation is that because there are several rehabilitation modalities employed in clinical practice (Table 3), it can be difficult to evaluate their actual effect on ICD duration. Further randomized controlled trials may be required.

There were some limitations to this study. First, only patients with PSP treated with ICD were enrolled, and those who received surgical intervention were excluded. The average systolic blood pressure was $128.67 \mathrm{mmHg}$, and there were no patients with tension pneumothorax, indicating that those in our study population did not have severe PSP. However, ICD treatment is an intervention that can be more easily implemented than surgical treatment, which can only be performed by a cardiovascular 
Table 4 Factors Associated with Duration of Intercostal Drainage Treatment in Patients with Primary Spontaneous Pneumothorax (PSP) by Univariate Linear Regression Analysis

\begin{tabular}{|l|l|l|l|}
\hline Factors & Coefficient & $\mathbf{9 5 \%}$ Confidence Interval & p value \\
\hline Age & 0.02 & $-0.11,0.17$ & 0.713 \\
Male sex & 0.86 & $-3.48,5.21$ & 0.692 \\
Smoking & 0.48 & $-3.40,4.36$ & 0.806 \\
Alcohol consumption & 2.78 & $-1.11,6.69$ & 0.158 \\
Recurrent PSP & 2.03 & $-2.28,6.36$ & 0.351 \\
Body mass index & -0.58 & $-1.28,0.11$ & 0.099 \\
Systolic blood pressure & 0.07 & $-0.03,0.17$ & 0.174 \\
Oxygen saturation & 0.41 & $-0.44,1.26$ & 0.341 \\
Serum albumin & 1.90 & $-6.34,10.15$ & 0.637 \\
Physical therapy modality & 2.78 & $-1.91,7.48$ & 0.240 \\
Visual analogue scale & 0.11 & $-0.51,7.30$ & 0.721 \\
\hline
\end{tabular}

Table 5 Factors Associated with Duration of Intercostal Drainage Treatment in Patients with Primary Spontaneous Pneumothorax (PSP) by Multivariate Linear Regression Analysis

\begin{tabular}{|l|l|l|l|}
\hline Factors & Coefficient & $\begin{array}{l}\text { 95\% Confidence } \\
\text { Interval }\end{array}$ & p value \\
\hline Body mass index & -1.38 & $-2.86,0.80$ & 0.063 \\
Systolic blood pressure & 0.21 & $0.01,0.42$ & 0.041 \\
Recurrent PSP & 7.69 & $0.43,14.95$ & 0.039 \\
\hline
\end{tabular}

thoracic surgeon. Second, the duration of recurrent PSP was not studied. Finally, some factors were not studied and some patients were excluded due to incomplete data, as this was a retrospective study.

PSP patients treated with ICD with a history of recurrence of PSP or high systolic blood pressure at presentation may require longer ICD duration and greater attention from the attending physician.

\section{Patient Consent}

This is not required due to retrospective data collection. Patient data confidentiality was not reported. The institutional review board of Khon Kaen University approved this human research study, which was performed in accordance with the Declaration of Helsinki.

\section{Acknowledgments}

The authors would like to thank Research Center in Back, Neck Other Joint Pain and Human Performance (BNOJPH), Khon Kaen University, Khon Kaen, Thailand.

\section{Funding}

There is no funding to report.

\section{Disclosure}

The authors report no conflicts of interest in this work.

\section{References}

1. Ashby M, Haug G, Mulcahy P, Ogden KJ, Jensen O, Walters JAE. Conservative versus interventional management for primary spontaneous pneumothorax in adults. Cochrane Database Syst Rev. 2014;12: CD010565.

2. Bense L, Eklund G, Wiman LG. Smoking and the increased risk of contracting spontaneous pneumothorax. Chest. 1987;92(6):1009-1012. doi:10.1378/chest.92.6.1009

3. Kaneko Y, Utsushikawa Y, Deguchi H, et al. Correlation with spontaneous pneumothorax and weather change, especially warm front approaching. J Thorac Dis. 2021;13(3):1584-1591. doi:10.21037/jtd-20-3395

4. Giles AE, Kidane B, Schellenberg M, Ball CG; Evidence Based Reviews in Surgery (EBRS) Group. The primary spontaneous pneumothorax trial: a critical appraisal from the surgeon's perspective. $J$ Thorac Cardiovasc Surg. 2021;162(5):1428-1432. doi:10.1016/j. jtcvs.2021.02.070

5. Olesen WH, Lindahl-Jacobsen R, Katballe N, et al. Recurrent primary spontaneous pneumothorax is common following chest tube and conservative treatment. World J Surg. 2016;40(9):2163-2170. doi:10.1007/s00268-016-3508-z

6. Bobbio A, Dechartres A, Bouam S, et al. Epidemiology of spontaneous pneumothorax: gender-related differences. Thorax. 2015;70 (7):653-658. doi:10.1136/thoraxjnl-2014-206577

7. Hallifax RJ, Goldacre R, Landray MJ, Rahman NM, Goldacre MJ. Trends in the incidence and recurrence of inpatient-treated spontaneous pneumothorax, 1968-2016. JAMA. 2018;320(14):1471-1480. doi:10.1001/jama.2018.14299

8. Brown SGA, Ball EL, Perrin K, et al. Conservative versus interventional treatment for spontaneous pneumothorax. $N$ Engl $J$ Med. 2020;382(5):405-415. doi:10.1056/NEJMoa1910775

9. Park H-S, Lee W-J, Kim Y-S. [The effects of deep breathing methods on pulmonary ventilatory function of pneumothorax patients undergoing a thoracotomy]. Taehan Kanho Hakhoe Chi. 2006;36(1):55-63. Ko. doi:10.4040/jkan.2006.36.1.55

10. Kim YR, Park SY. The effects of the systematic breathing exercises program on recovery of patients with pneumothorax. J Korean Crit Care Nurs. 2012;5(2):28-36.

11. Saccò M, Meschi M, Regolisti G, et al. The relationship between blood pressure and pain. J Clin Hypertens. 2013;15(8):600-605. doi: $10.1111 /$ jch. 12145 
12. Vuong NL, Elshafay A, Thao LP, et al. Efficacy of treatments in primary spontaneous pneumothorax: a systematic review and network meta-analysis of randomized clinical trials. Respir Med. 2018;137:152-166. doi:10.1016/j.rmed.2018.03.009

13. Kepka S, Dalphin JC, Parmentier AL, et al. Primary spontaneous pneumothorax admitted in emergency unit: does first episode differ from recurrence? A Cross-Sectional Study. Can Respir J. 2017;2017:2729548. doi:10.1155/2017/2729548
14. Saad AB, Migaou A, Ammar M, et al. [Recurrence score to predict the risk of recurrence after first episode of primary spontaneous pneumothorax]. Pan Afr Med J. 2020;36:107. Frence. doi:10.11604/ pamj.2020.36.107.23432

\section{Publish your work in this journal}

The Open Access Emergency Medicine is an international, peerreviewed, open access journal publishing original research, reports, editorials, reviews and commentaries on all aspects of emergency medicine. The manuscript management system is completely online

Submit your manuscript here: https://www.dovepress.com/open-access-emergency-medicine-journal and includes a very quick and fair peer-review system, which is all easy to use. Visit http://www.dovepress.com/testimonials.php to read real quotes from published authors. 